\title{
Unified Scattering Parameters formalism in terms of Coupled- Mode Theory for investigating hybrid single-mode/two-mode photonic interconnects
}

\author{
Yann G. Boucher ${ }^{1-2,{ }^{*}}$, Alberto Parini ${ }^{1}$, and Patrice Féron ${ }^{1}$ \\ ${ }^{1}$ FOTON, Systèmes Photoniques, UMR CNRS 6082, Université de Rennes 1, 22305 Lannion, France \\ ${ }^{2}$ ENIB, Technopôle Brest-Iroise, CS 73862, 29638 Brest, France
}

\begin{abstract}
In terms of Linear Algebra, a directional coupler between a single-mode waveguide and a twomode waveguide can be thought of as formally equivalent to a set of three mutually coupled single-mode waveguides. Its responses, easily derived in the frame of ternary Coupled-Mode Theory, are used to establish analytically the scattering parameters of a hybrid ring-based modal multiplexer.
\end{abstract}

\section{Introduction}

Mode-division multiplexing (MDM), also referred to as Spatial-division multiplexing (SDM), relies on multimode waveguides to simultaneously transmit signals allocated on different spatial modes, all sharing the same wavelength [1-3]. In the single-mode domain, the system-level dimensioning of optical multi-port devices is easily carried out by means of the powerful formalism of Scattering Parameters, in which each output wave is simply expressed as a linear combination of all possible input waves. The exchange of energy between modes takes place in directional couplers through an overlap of their evanescent part, a mechanism well described in terms of Coupled-Mode Theory (CMT) [4-5]. There is no fundamental reason why, in the multi-mode domain, this same analytical tool shouldn't appear as relevant.

In what follows, we investigate most specifically the key element for hybrid multimode integrated structures: an asymmetric directional coupler between a singlemode waveguide (SMWG) and a two-mode waveguide (TMWG). Taking advantage of the formal equivalence between a multi-mode waveguide and a set of mutually coupled single-mode ones [6-7], we first derive its responses (transmittance and coupling), before inserting the structure into a more complex system, a ring-based modal multiplexer, for which we establish the scattering parameters under a closed form.

\section{Single-mode to multimode coupling}

Consider an asymmetric coupler between a SMWG and a TMWG (Fig. 1). The latter is dimensioned in order to support a fundamental guided mode with even symmetry and a first-order mode with odd symmetry, both characterized by their respective propagation constants $\beta_{\text {even }}$ and $\beta_{\text {odd }}$. Time dependence is taken as $\exp (+i \omega t)$.

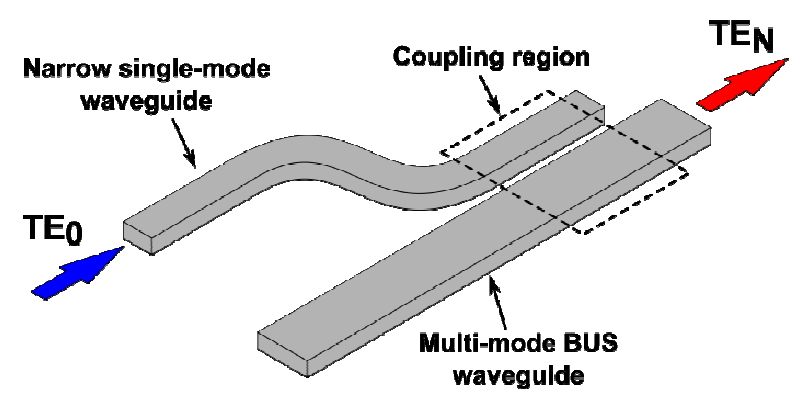

Fig. 1. Typical coupling between a SMWG and a TMWG.

Taking advantage of the formal equivalence between the TMWG and a set of two mutually coupled SMWGs [6], we describe the coupling region as a ternary coupler of length $d$ [7], as schematically depicted in Fig. 2.

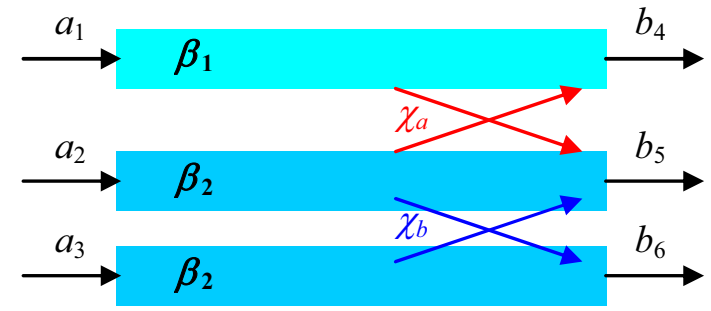

Fig. 2. Modelling of the directional coupler between the upper SMWG and the lower TMWG (itself seen as a set of 2 coupled SMWGs) as a six-port network; $a_{i}=F_{i}(0), b_{j}=F_{j}(d)$.

In this basis, the evolution operator (along the common $z$-axis) reads:

$$
i \frac{\partial}{\partial z}\left(\begin{array}{l}
F_{1} \\
F_{2} \\
F_{3}
\end{array}\right)=\left(\begin{array}{ccc}
\beta_{1} & \chi_{a} & 0 \\
\chi_{a} & \beta_{2} & \chi_{b} \\
0 & \chi_{b} & \beta_{2}
\end{array}\right)\left(\begin{array}{l}
F_{1} \\
F_{2} \\
F_{3}
\end{array}\right)
$$

where $F_{n}$ is the complex amplitude of "mode" $\mathrm{N}^{\circ} n$, 


$$
\beta_{2}=\frac{\beta_{\text {even }}+\beta_{\text {odd }}}{2}, \quad \chi_{b}=\frac{\beta_{\text {even }}-\beta_{\text {odd }}}{2} .
$$

The average propagation constant $\beta$, the mismatch $\Delta$ and the effective coupling constant $\chi$ are defined as:

$$
\beta=\frac{\beta_{1}+2 \beta_{2}}{3}, \quad \Delta=\frac{\beta_{1}-\beta_{2}}{3}, \quad \chi=\sqrt{\chi_{a}^{2}+\chi_{b}^{2}} .
$$

Working in terms of Slowly Varying Envelope Amplitudes $A_{n}$ such as $F_{n}=A_{n} \mathrm{e}^{-i \beta z}$, we get:

$$
i \frac{\partial}{\partial z}\left(\begin{array}{l}
A_{1} \\
A_{2} \\
A_{3}
\end{array}\right)=\chi\left(\begin{array}{ccc}
2 \delta & \sin \alpha & 0 \\
\sin \alpha & -\delta & \cos \alpha \\
0 & \cos \alpha & -\delta
\end{array}\right)\left(\begin{array}{l}
A_{1} \\
A_{2} \\
A_{3}
\end{array}\right)
$$

with $\delta=\Delta / \chi$ the reduced mismatch and $\alpha=\operatorname{atan}\left(\chi_{a} / \chi_{b}\right)$. Eigenvalues and eigenvectors of the reduced operator are obtained through a standard diagonalization procedure (involving Cardano's method for solving the third-order characteristic equation), leading to a complete determination of the three ternary super-modes (respectively slow, "neutral" and fast). We revert eventually to the even/odd TMWG basis, with:

$$
\begin{aligned}
& \left(\begin{array}{l}
b_{\text {even }} \\
b_{\text {odd }}
\end{array}\right)=\frac{1}{\sqrt{2}}\left(\begin{array}{cc}
1 & 1 \\
-1 & 1
\end{array}\right)\left(\begin{array}{l}
b_{5} \\
b_{6}
\end{array}\right), \\
& \left(\begin{array}{l}
a_{2} \\
a_{3}
\end{array}\right)=\frac{1}{\sqrt{2}}\left(\begin{array}{cc}
1 & -1 \\
1 & 1
\end{array}\right)\left(\begin{array}{l}
a_{\text {even }} \\
a_{\text {odd }}
\end{array}\right),
\end{aligned}
$$

then we derive analytically the scattering matrix of the coupler, expressed under the following form:

$$
\left(\begin{array}{c}
b_{4} \\
b_{\text {even }} \\
b_{\text {odd }}
\end{array}\right)=\left(\begin{array}{ccc}
t_{s} & k_{s / e} & k_{s / o} \\
k_{s / e} & t_{e} & k_{\text {e/o }} \\
k_{s / o} & k_{\text {e/o }} & t_{o}
\end{array}\right)\left(\begin{array}{c}
a_{1} \\
a_{\text {even }} \\
a_{\text {odd }}
\end{array}\right) .
$$

Because of the symmetries, only six parameters are involved: three "direct transmissions" $\left(t_{s}, t_{e}, t_{o}\right)$ and three "cross-couplings" $\left(k_{s / e}, k_{s / o}, k_{e / o}\right)$. For the sake of illustration, let us consider the case when perfect phasematching is achieved between the upper SMWG and the odd mode of the lower TMWG:

$$
\beta_{1}=\beta_{o d d}=\beta_{2}-\chi_{b} \Rightarrow \delta=\frac{-\cos \alpha}{3} .
$$

The power exchange between the modes is depicted in Fig. 3 as a function of the dimensionless coupling strength $\chi d$, integrated over the interaction length $d$, when only the upper single-mode (1) is present at the input. Up to $96 \%$ of the power can be coupled into the lower odd mode $(o)$. Note that residual coupling into the lower even mode $(e)$ also occurs. For comparison, the dashed line represents the result of a much more simplistic two-mode coupling approach, neglecting the presence of the third $(e)$ mode.

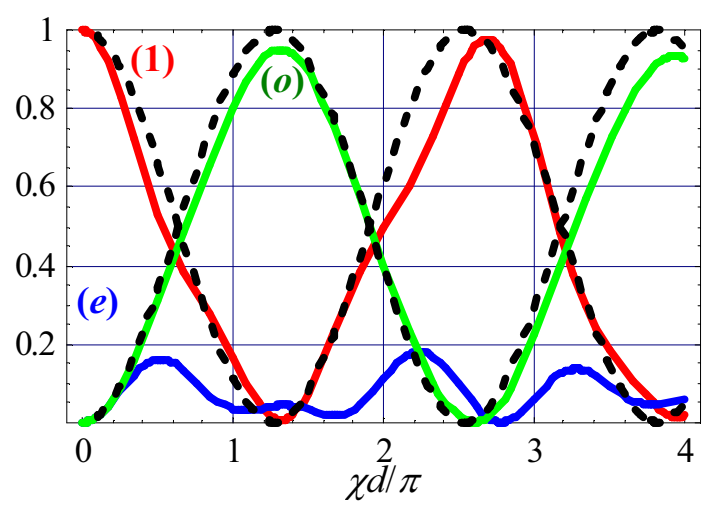

Fig. 3. Power exchange as a function of the coupling from input $a_{1}$ to outputs $b_{1}, b_{\text {even }}, b_{\text {odd }}$. In dashed line, the result of a much more simplistic approach with only two-mode coupling, neglecting the residual crosstalk into the mismatched $(e)$ mode.

We would like to emphasize that the calculations are carried out under a reduced form, the only assumption being that of eqn.(7); this means that the results carry a universal significance for any "single to odd" phasematched directional coupler, whatever the precise optogeometric parameters of the actual device.

Let us now investigate a more elaborate device: a ring-based modal multiplexer.

\section{Ring-based modal multiplexer}

The system under consideration [Fig. 4] is made of an input SMWG connected to an output TMWG through a ring resonator $(\mathrm{RR})$ in the "racetrack" configuration. For the sake of clarity, let us assume that the SMWG and the RR share the same propagation constant $\beta_{1}$. The upper binary coupler, of length $d$, is completely defined by its own scattering parameters: direct and cross-transmission $t_{1}$ and $k_{1}$, respectively. The lower ternary coupler, also of length $d$, is described as seen above. The whole device works in a way similar to a single-mode add-drop filter, except for the additional feature of mode conversion.

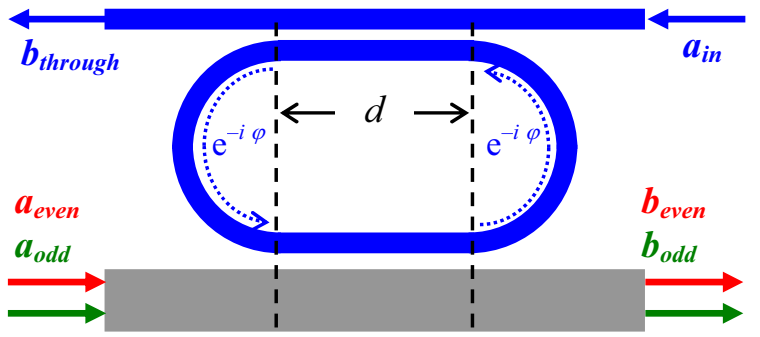

Fig. 4. Ring-based modal multiplexer seen as a multi-port network. All ports (with their phase reference) are actually located at each end of the couplers.

Once again, we take advantage of Linear Algebra in order to express each relevant output field $\left(b_{\text {through }}, b_{\text {even }}\right.$, $\left.b_{o d d}\right)$ as a linear combination of all input fields $\left(a_{i n}, a_{\text {even }}\right.$, $\left.a_{o d d}\right)$. Once closed the feedback loop provided by the RR, a straightforward self-consistent calculation leads eventually to the following analytical expressions: 


$$
\begin{gathered}
b_{\text {even }}=a_{\text {in }} \frac{k_{1} k_{s / e} \mathrm{e}^{-i \varphi}}{D}+ \\
a_{\text {even }}\left(t_{e}+\frac{t_{1} k_{s / e}^{2} \mathrm{e}^{-2 i \varphi}}{D}\right)+ \\
a_{\text {odd }}\left(t_{o}+\frac{t_{1} k_{s / o}^{2} \mathrm{e}^{-2 i \varphi}}{D}\right), \\
b_{\text {odd }}=a_{\text {in }} \frac{k_{1} k_{s / o} \mathrm{e}^{-i \varphi}+}{D}+ \\
a_{\text {even }}\left(k_{\text {e/o }}+\frac{t_{1} k_{s / e} k_{s / o} \mathrm{e}^{-2 i \varphi}}{D}\right)+ \\
a_{\text {odd }}\left(t_{o}+\frac{t_{1} k_{s / o}^{2} \mathrm{e}^{-2 i \varphi}}{D}\right), \\
b_{\text {through }}=a_{\text {in }}\left(t_{1}+\frac{k_{1}^{2} t_{s} \mathrm{e}^{-2 i \varphi}}{D}\right)+ \\
a_{\text {even }}\left(\frac{k_{1} k_{s / e} \mathrm{e}^{-i \varphi}}{D}\right)+ \\
a_{\text {odd }}\left(\frac{k_{1} k_{s / o} \mathrm{e}^{-i \varphi}}{D}\right)
\end{gathered}
$$

with $D$ the unmistakable signature of a feedback loop:

$$
D=1-t_{1} t_{s} \mathrm{e}^{-2 i \varphi}
$$

where $\varphi$ denotes the phase change through a half-circle inside the RR (from the end of the upper coupler to the entrance of the lower one, or vice versa). The complex quantity $t_{1} t_{s} \mathrm{e}^{-2 i \varphi}$ represents the amplitude and phase change over a round-trip, experienced by a wave circulating in the RR.

The overall form taken by scattering parameters is worth some comments. From eqn.(8), one can see that they fall into one of two categories. The simplest ones (from $a_{\text {in }}$ to $b_{\text {even }}$ or to $b_{\text {odd }}$, from $a_{\text {even }}$ or $a_{\text {odd }}$ to $b_{\text {through }}$ ) take the form of a single-pass numerator over the common denominator $D$. The other ones come as the sum of two terms, expressing the interference between two possible paths: for instance, the transmittance from $a_{i n}$ to $b_{\text {through }}$ results from the competition between the direct path through the upper coupler $\left(t_{1}\right)$, and the path through the $\operatorname{RR}\left(k_{1}^{2} t_{s} \mathrm{e}^{-2 i \varphi}\right)$, itself amplified by the feedback loop $(D)$.

It is therefore possible to exploit these interference effects in order to fine-tune the parameters of the whole system. For instance, by equating the internal transmission coefficients $t_{1}$ and $t_{s}$ on both couplers seen from inside the RR, it becomes possible (by destructive interference) to exactly cancel the transmittance from $a_{i n}$ to $b_{\text {through }}$, thus converting all the power of the upper single-mode input into the output modes of the lower TMWG. Note, however, that such a mechanism relies on interference: in practice, we have to make sure that the working wavelength is actually attuned to a resonance.
Tolerance and bandwidth issues have to be kept in mind, but will not be considered further in the present work.

Besides, it can be interesting to note that, once established the scattering parameters of both couplers, expressions (8), themselves closely related to the topology of the network, could also be recovered from graph theory considerations [8].

\section{Conclusions}

We have presented a modelling technique based on scattering matrix formalism adapted to multi-mode devices. By exploiting the formal identity between a two-mode waveguide and a set of two mutually coupled single-mode waveguides, the multi-mode interaction is treated within the usual framework of Coupled Mode Theory (CMT). This approach has been tested on the typical example of a ring-based modal multiplexer, opening the way to a systematic exploration of the parameter space for the purpose of design. Taking into account possible losses in the waveguides would be straightforward.

This work is supported by the Labex CominLabs (French National Research Agency program "Investing for the Future" ANR-10-LABX-07-01) within the "3D-Optical-ManyCores" Project (http://www.3d-opt-many-cores.cominlabs.ueb.eu/). The authors would also like to thank Prof. Christophe Peucheret (FOTON Sys.Phot.) for helpful and stimulating discussions.

\section{References}

1. Y. Ding, J. Xu, F. Da Ros, B. Huang, H. Ou and C. Peucheret, Opt. Express, 21, 8, 10376-82 (2013)

2. D. Dai, J.E. Bowers, Nanophotonics, 3, 4-5, 283311 (2014)

3. C. Peucheret, Y. Ding, J. Xu, F. Da Ros, A. Parini, H. Ou, Advanced Photonics 2015 OSA Technical Digest (online), paper SpT2E.3 (2015)

4. A. Yariv \& P. Yeh, Optical Waves in Crystals (Wiley, New York, 1984)

5. T. Tamir (Ed.), Guided-Wave Optoelectronics (Springer-Verlag, Berlin, 1990)

6. Y.G. Boucher, Opt. Eng., 53, 7, 071810 (2014)

7. Y.G. Boucher, A. Parini, P. Féron, Optical Quantum Electron. (to be published)

8. E.J. Henley \& R.A. Williams, Graph Theory in Modern Engineering (Academic Press, New York and London, 1973) 